\title{
Inhibition of Hyperprogressive Cancer Disease Induced by Immune-Checkpoint Blockade Upon Co- Treatment with Meta-Tyrosine and p38 Pathway Inhibitor.
}

\section{Daniela Romina Montagna ( $\nabla$ daniela.r.montagna@gmail.com )}

Laboratory of Experimental Oncology, Instituto de Medicina Experimental (IMEX-CONICET), Academia Nacional de Medicina de Buenos Aires

\section{Alejandra Duarte}

Laboratory of Experimental Immunology, IMEX-CONICET, Academia Nacional de Medicina de Buenos Aires

\section{Paula Chiarella}

Laboratory of Experimental Oncology, Instituto de Medicina Experimental (IMEX-CONICET), Academia Nacional de Medicina de Buenos Aires

\section{Barbara Rearte}

Laboratory of Physiology of Inflammatory Processes, IMEX-CONICET, Academia Nacional de Medicina de Buenos Aires

\section{Oscar Bustuoabad}

Retired, Buenos Aires, Argentina

\section{Mónica Vermeulen}

Laboratory of Antigen Presenting Cells and Inflammatory Response, IMEX-CONICET, Academia Nacional de Medicina de Buenos Aires

\section{Raúl Ruggiero}

Laboratory of Experimental Oncology, Instituto de Medicina Experimental (IMEX-CONICET), Academia Nacional de Medicina de Buenos Aires

\section{Research Article}

Keywords: hyperprogressive cancer disease, immune checkpoints inhibitors, meta-tyrosine, metastases, murine tumors.

Posted Date: November 17th, 2021

DOl: https://doi.org/10.21203/rs.3.rs-969535/v1 
License: (c) (i) This work is licensed under a Creative Commons Attribution 4.0 International License. Read Full License 


\section{Abstract \\ Background}

Although immune-checkpoint inhibitors $(\mathrm{ICl})$ are overall promissory for cancer treatment, they entail, in some cases, an undesired side-effect called hyperprogressive-cancer disease (HPD) associated with acceleration of tumor growth and shortened survival.

\section{Methods}

To understand the mechanisms of HPD we assayed the ICI therapy on two murine tumors widely different regarding immunogenicity and, subsequently, on models of local recurrences and metastases of these tumors. To potentiate the immune response (IR), we combined ICI with meta-tyrosine - that counteracts immune-suppressive signals - and a selective inhibitor of p38 pathway that proved to counteract the phenomenon of tumor-immunostimulation.

\section{Results}

ICI were therapeutically effective against both tumor models (proportionally to their immunogenicity) but only when they faced incipient tumors. In contrast, ICI produced acceleration of large and residual tumors. The combined treatment strongly inhibited the growth of large tumors and it managed to cure $80 \%$ of mice with local recurrences and $60 \%$ of mice bearing residual metastases.

\section{Conclusions}

Tumor enhancement was paradoxically correlated to a weak increase of the antitumor IR suggesting that a weak IR - different from a strong tumor-inhibitory one - may produce stimulation of tumor growth, mimicking the HPD observed in some clinical settings.

\section{Background}

In the last 20 years, different novel immunological strategies were developed to enhance the basal antitumor immune response evoked by growing tumors as well as to counteract the emergence of tumorassociated negative immune-regulatory mechanisms that could down-regulate such response (1-4).

Among these strategies, blockade of immune-checkpoints has been considered the most promising one and a potential revolution for the treatment of clinical cancer (5).

This hopeful expectation is based on a large body of evidence that demonstrates that experimental growing murine tumors may be inhibited or even eradicated upon treatment with inhibitors of the 
cytotoxic T-lymphocyte-associated protein (CTLA)-4 and the programmed cell death-1 (PD-1)/PD ligand1(PD-L1) pathway.

These treatments have also increased the overall survival and progression-free survival of humans affected by some cancers such as melanoma and non-small cell lung, colo-rectal, and renal cell carcinoma (6-8). However, some treated patients show no improvement and a variable fraction of them exhibits a condition called 'hyperprogressive cancer disease' (HPD) associated with sharp acceleration of tumor growth, worse prognosis, and shortened survival times (9-11). In addition, some initial responders eventually develop resistance to therapy, and others may be afflicted by immune-related adverse events (12).

Two main arguments could be invoked to explain the disparity between the resounding successes achieved in experimental models and the more modest results observed in clinical settings.

The first one is associated with the fact that experimental models are usually strongly immunogenic chemically-induced tumors while, in contrast, most human tumors may exhibit significantly lower immunogenicity. In support of this contention, murine tumors of spontaneous origin - which have been considered the best models for common human cancers - usually exhibit weak or undetectable immunogenicity (13-15).

The second argument is related to the fact that immune-checkpoint inhibitors (ICI) were truly effective in restraining experimental murine tumors, although only when they faced incipient tumors. Afterwards, as the tumor becomes larger, and resembles the size that could usually be detectable at first clinical inspection, null, or even stimulatory effects on tumor growth have been observed, especially when large tumors were concerned (16).

It could be argued that immunologic strategies are second-line therapies after surgery, radiotherapy, or chemotherapy have reduced tumor burden. In such cases, residual tumors associated with local recurrences or metastases may be made up of a small number of tumor cells that would mimic an incipient tumor against which $\mathrm{ICl}$ proved to be effective. However, despite their similarity concerning the number of cells, both incipient and residual tumors might behave utterly different in terms of their sensitivity to immunologic treatments. In effect, cells from incipient tumors are starting to grow in an otherwise healthy host while cells from a residual tumor are placed in a microenvironment that could have been drastically altered by the previous presence of a tumor.

If the arguments above mentioned were valid, the use of appropriate tumor models that closely resemble the real clinical situation might be helpful to understand - in controlled conditions - both the scope and the limitations of these anti-tumor immunologic treatments (17).

This paper has been designed:

a. To evaluate the efficacy of the blockade of CTLA-4 and PD-L1 immune checkpoint - and also the therapy with antitumor vaccines - in function of target immunogenicity. To this aim, we have 
characterized and utilized two murine tumors, a strongly immunogenic methylcholanthrene-induced fibrosarcoma (similar to those currently used to assay immunological therapies in laboratory animals) and a weakly immunogenic and highly metastatic mammary carcinoma of spontaneous origin.

b. To determine whether or not the inhibitory effect observed on incipient tumors upon ICl therapy is a good predictor of the outcome of this therapy on residual tumors. In this way, we have compared the efficacy of $\mathrm{ICl}$ on the growth of incipient, mid-, and large-sized tumors with that on the re-growth of local tumor recurrences and metastases after surgical tumor removal.

c. To elucidate the mechanisms underlying the accelerating effect (HPD) observed on large tumors upon ICl therapy. The phenomenon of HPD upon ICl suggests that other points of immunologic control and/or aspects not accurately explored of the antitumor immune response might play a central role in limiting the efficacy of this therapy. To account for these possibilities we have, in the first place, used meta-tyrosine ( $m$-Tyr), an unnatural isomer of tyrosine that it has recently been demonstrated to be capable of rescuing the organism from states of immunosuppression by mechanisms different from the known ICI (18). In the second place, we have considered the possibility that the antitumor immune response may be not linear but biphasic with strong immune responses producing inhibition while weak ones inducing stimulating effects on tumor growth (Suppl. Figure 1) $(16,19,20)$. In a former work (16), we have suggested that a "weak" antitumor immune response associated with some stages of tumor growth enhanced the production of chemokines aimed to recruit macrophages at the tumor site, which, upon activation of TLR4 and p38 signaling pathways, would produce growth-stimulating signals leading to an accelerated tumor growth. Based on the above considerations, we have combined herein the blockade of CTLA-4 and PD-1/PD-L1 immune-checkpoints with both $\mathrm{m}$-Tyr - to boost the antitumor immune response by counteracting other points of immunologic control - and inhibitors of p38 pathway - to counteract the phenomenon of tumor immunostimulation - with the hope of enabling otherwise indolent or tumor-promoting schedules to produce inhibition of tumor growth.

\section{Materials And Methods}

\section{Animals}

Female and male BALB/c mice were bred in the Academia Nacional de Medicina de Buenos Aires facilities. They were used at 2-3 months of age and 20-25 grams of weight. Nude BALB/c mice and NOD Scid Gamma (NSG) mice were purchased from Comisión Nacional de Energía Atómica and Instituto de Biología y Medicina Experimental, Argentina, respectively. Care of mice, an early experimental endpoint and all methods were according to NIH Guide for the Care and Use of Laboratory Animals. All experimental protocols were approved by the Committee for the Care and Use of Laboratory Animals (CICUAL) of Instituto de Medicina Experimental (IMEX-CONICET, protocol N005/15). All methods were 
performed in accordance with ARRIVE guidelines. Randomization was carried out by Rand() in software Excel and in vivo experiments were blinded.

\section{Murine Tumors}

LMM3 : highly metastatic mammary carcinoma, kindly provided by Dr. L. Colombo (Instituto Angel Roffo, Buenos Aires, Argentina).

MC-C: strongly immunogenic fibrosarcoma induced by the chemical 3-methylcholanthrene.

More details of tumor models and surgical procedures have been reported previously $(1,21-23)$. Tumor dose 50 (TD50): number of tumor cells able to grow in $50 \%$ of mice. Tumor volume was calculated as $0.4 a b^{2}$, where $a$ and $b$ are the larger and smaller diameters, respectively $(21,22)$. We defined incipient, mid-sized, and large-sized tumors, those whose volumes were $\leq 10 \mathrm{~mm}^{3}, 100-400 \mathrm{~mm}^{3}$, and $>500 \mathrm{~mm}^{3}$, respectively. The medium was RPMI 1640 (Gibco) supplemented as described (1). Tumor lysates, bone marrow-derived dendritic cells isolation, splenocytes isolation, and histological analyses were performed as previously reported $(1,24,25)$. Tumor growth rate (TGR) was expressed as the increase of tumor volume per unit time $=$ tumor volume at day $B$ - tumor volume at day $A / B-A(9-11)$.

\section{Reagents}

HGMB1 and HSP60 were quantified using ELISA kits from Pepro-Tech, following the manufacturer's recommendations. TNF- $a$, IL-12p70, IL-10 and TGF- $ß$ were quantified using ELISA kits from R\&D Systems.

\section{Tumor vaccination strategies}

Pre-treatment with X-lethally irradiated (LI) tumor cells and pre-treatment with dendritic cells (DC) incubated with tumor lysate were carried out as reported (1, 25-27).

\section{Drugs, ICl and radiotherapy}

DL-m-tyrosine (Sigma-Aldrich) and p38 inhibitor SB202190 (Santa Cruz Biotechnology) were used as described $(18,28)$. JSI-124 (Indofine Chemical Company, Hillsborough, NJ), an inhibitor of STAT3; JQ1 (Sigma-Aldrich), an inhibitor of PD-L1; blocking anti-mouse PD-L1, clone 10F.9G2 and anti-mouse CTLA-4 (CD152), clone 9H10 (BioXCell), were used as reported (29-31). Treatments with vincristine and radiotherapy (2000 grades in the tumor area; Philips 250/15 radiotherapy device at $220 \mathrm{Kv}, 14 \mathrm{~mA}$ ) were used as described (1).

\section{Flow cytometry}

Dendritic cells were incubated with different combinations of the mAbs: anti-CD11c, anti-IAd (MHC class II), anti-CD86 and anti-CD80, following the manufacturer's recommendations. Splenocytes, and/or tumor cells were incubated with antibodies: anti CD3, CD4, CD8, CD11b, PD-1, PD-L1 - clone MIH5 -, phosphorylated-STAT3 (pSTAT3) from Ap-Biotech, Argentina, and 5,6 Carboxi Fluorescein diacetate Succinimidyl Ester (CFSE) from Molecular Probes; Eugene, OR, USA. Fluorescence of individual cells was 
measured in a flow cytometer (Becton Dickinson) and was analyzed by Flowing (Software version 2.5.1, Turku Centre for Biotechnology. University of Turku, Finland). More details were given elsewhere (1).

\section{Proliferation assays}

Lymphocyte proliferation was evaluated by CFSE staining (Molecular Probes). Briefly, $1 \times 10^{7} \mathrm{cell} / \mathrm{s} / \mathrm{ml}$ were suspended in $0.3 \%$ BSA/PBS. Then, $1 \mu \mathrm{l}$ of CFSE was added for each $\mathrm{ml}(0.5 \mu \mathrm{M})$ (Invitrogen) and cells were incubated for $15 \mathrm{~min}$ at $37^{\circ} \mathrm{C}$. Cells were washed three times with complete RPMI and incubated for $5 \mathrm{~min}$ at $37^{\circ} \mathrm{C}$ between washes. Afterward, $1 \times 10^{5}$ lymphocytes were cultured in 96 -well flat-bottom plates for 24,48 , or $72 \mathrm{~h}$ in the presence or absence of $3 \times 10^{3} \mathrm{DC}$ pre-treated with tumor lysates and/or $\mathrm{m}$-Tyr. Then, 30000 events were collected and CFSE low expression (proliferating lymphocytes, FL-1) was analyzed by flow cytometry as described above.

\section{Western blotting}

Western blotting was carried out with standard techniques as described and analyzed by ImageQuant software. The following antibodies were used: anti-p-STAT3, anti-STAT3 (Santa Cruz Biotechnology, Paso Robles, CA), anti-p38 (Santa Cruz Biotechnology) and anti- $\beta$-actin (Cell Signaling Technology, Danvers, MA). Levels of each band were normalized with $\beta$ actin densitometry units as reported (16).

\section{Statistical analysis}

Student's t-test, ANOVA, Mann Whitney U test and Kaplan-Meier estimator for survival curves were used. Values were expressed as mean \pm standard error (SE). Differences were considered to be significant whenever the $P$-value was 0.05 or smaller.

\section{Results}

\section{Different properties of tumor models and preventive immunologic strategies}

MC-C tumor proved to be strongly immunogenic as far as pre-treatment of mice with lethally-irradiated MC-C tumor cells or with DC stimulated with MC-C tumor lysate strongly prevented the growth of live MCC tumor cells implanted thereafter (Figure 1A). This effect was tumor-specific and T-cell dependent (not shown), indicating specific and robust tumor antigens.

MC-C tumor immunogenicity was associated with the capacity of $\mathrm{MC}-\mathrm{C}$ tumor lysate to promote the maturation of DC as evaluated by the up-regulation of cell surface receptors CD80, CD86 and MHC II as well as production of the inflammatory cytokines TNF- $a$ and IL-12p70 (Figure 1B, C and D). In addition, this capacity of MC-C tumor lysate was correlated to its high concentration of HGMB1 and Hsp60 - two recognized danger signals that favor DC maturation - and low concentration of IL-10 and TGF- $\beta$ - two known immunosuppressive cytokines that inhibit DC maturation (1, 24); (Figure 1E and F). In turn, MC-C tumor cells exhibited low constitutive expression of phosphorylated STAT3 (pSTAT3) - an activated 
molecule that is involved in the transcription of genes that induce tolerogenic and immunosuppressive signals (16); (Figure 2A, B and C) - and low expression of surface PD-L1 (Figure 2D, E, and G).

On the other hand, LMM3 tumor displayed weak (if any) immunogenicity (Figure 1A), which was associated with the incapacity of LMM3 tumor lysate to promote maturation of DC (Figure 1B, C and D). This incapacity was correlated to a low concentration of HGMB1 and Hsp60 and a high concentration of IL-10 and TGF- $\beta$ present in LMM3-tumor lysate (Figure 1E and F). LMM3 tumor cells exhibited high constitutive expression of pSTAT3 (Figure 2A, B and C) and high expression of surface PD-L1 (Figure 2D, E and $H)$.

The immunosuppressive properties of LMM3 tumor lysate were confirmed by its ability to counteract the capacity of LPS to promote DC maturation. In contrast, lysates prepared from normal spleen cells or MCC tumor cells did not counteract LPS capacity (Suppl. Figure 2).

Tumor lysate prepared from LMM3 tumor cells pre-treated with a natural inhibitor of pSTAT3 called JSI124 acquired a significant capacity to promote the maturation of DC in a dose-dependent manner (Figure 2I). In turn, pre-treatment of mice with these DC produced a significant protective effect (preventive vaccination) against the growth of live tumor cells implanted thereafter (Figure $2 \mathrm{~J}$ ). A vaccinating similar effect was achieved with lethally-irradiated LMM3 tumor cells that had been pre-treated - before being irradiated - either with JQ1 (to inhibit the expression of PD-L1 or JSI-124 (to inactivate PSTAT3). These protective effects were tumor-specific and T-cell dependent (not shown).

The above considerations suggest that both chemically-induced MC-C and spontaneous LMM3 tumors bear specific antigens. However, in LMM3, these antigens seemed to be hidden by immunosuppressive signals released by the own tumor cells. It is worth noting that, even counteracting the mechanisms that prevent the onset of an anti-LMM3 tumor immune response, the magnitude of both the maturation of DC by LMM3 lysate and the preventive vaccinations was always several orders lower than that achieved with $\mathrm{MC}-\mathrm{C}$ tumor. It suggests that the strength of MC-C tumor antigens is much greater than that of LMM3 ones.

\section{Contrasting effects of immunotherapies on growing tumors}

When MC-C tumor cells were inoculated in naïve mice, tumor-bearing mice produced a significant antitumor immune response (although not strong enough to inhibit the growing tumor) characterized by classical markers of anti-tumor immunity (Suppl. Figure 3). This significant immune response was, in turn, correlated to a) a relatively low expression of PD-L1 on the surface of tumor-infiltrating CD11 $\mathrm{b}^{+}$ myeloid cells (Figure $2 \mathrm{~F}$ and $\mathrm{G}$ ) and $b$ ) low expression of PD- 1 in the surface of both T CD ${ }^{+}$and CD $4^{+}$ splenic lymphocytes (Suppl. Figure 4).

On the other hand, when LMM3 tumor cells were inoculated in naïve mice, tumor-bearing mice produced a weak (if any) anti-tumor immune response (Suppl. Figure 3), which was correlated to a) high expression 
of PD-L1 on the surface of tumor-infiltrating $\mathrm{CD} 11 \mathrm{~b}^{+}$myeloid cells (Figure $2 \mathrm{~F}$ and $\mathrm{H}$ ) and $b$ ) increased expression of PD- 1 in the surface of both $\mathrm{T} \mathrm{CD} 8^{+}$and $\mathrm{CD} 4^{+}$splenic lymphocytes (Suppl. Figure 4).

Based on these observations and on the fact that LMM3 tumor cells displayed a significantly higher expression of PD-L1 than MC-C tumor cells (see above Figure 2D, E, G and H), it was expected that the blockade of the PD-1/PD-L1 pathway might be more useful to treat LMM3 than MC-C growing tumors by unlocking the onset of an otherwise almost inexistent anti-tumor immune response. On the other hand, the blockade of CTLA-4 might be more beneficial to treat MC-C than LMM3 growing tumors by enhancing an ongoing anti-tumor immune response. Our experiments confirmed these expectations. However, in both models, the combined treatment with anti-CTLA-4 and anti-PD-L1 was better than each separately.

In the MC-C tumor model, vaccines based on lethally-irradiated MC-C tumor cells or DC stimulated by MC$C$ tumor lysate produced inhibitory effects on growing $M C-C$ tumors somewhat similar to those achieved with ICl (Figure 3A and B).

On the other hand, no impact on growing LMM3 tumors was obtained with lethally-irradiated LMM3 tumor cells or DC stimulated with LMM3 lysate. Only the use of DC stimulated with LMM3 lysate prepared with inactivated STAT3 produced some anti-tumor effect although lower than that attained with $\mathrm{ICl}$ in both subcutaneous tumors and lung metastases (Figure $3 \mathrm{C}, \mathrm{D}$ and $\mathrm{E}$ ).

In summary, as shown comparatively in Figures $3 A-D$, the immunologic-mediated antitumor effects were stronger on the strongly immunogenic tumor (MC-C) than on the weakly immunogenic one (LMM3).

It is worth noting that the striking success of all of these immunologic treatments was achieved against incipient ( $\leq 10 \mathrm{~mm}^{3}$ ) but not larger growing tumors. In fact, in mice bearing large-sized tumors (mean volume: $650-800 \mathrm{~mm}^{3}$ ) - either strongly or weakly immunogenic - these treatments not only did not produce any inhibitory effect but an enhanced tumor growth (Figure 3A - D) while no effect was observed on metastases (Figure 3E). Attempting to get an effective treatment against these tumors, we doubled the doses of ICl. However, the results were worse than before since, upon this double dose treatment, all mice showed severe manifestations of auto-immunity and reached the experimental endpoint rapidly after the last dose (not shown).

\section{Contrasting outcomes of immunological strategies on incipient and residual tumors}

Although inefficient to inhibit mid- and large-sized tumors, the ability of $\mathrm{ICl}$ to restrain the growth of incipient tumors might still have great clinical potential value if it were demonstrated that residual tumors- supposedly the targets of $\mathrm{ICl}$ in clinical settings - would behave in the same way as incipient tumors regarding their sensitivity to immunologic treatments. To test this contention, we used two clinically relevant tumor models: 
a. A model of local recurrence after subcutaneous (s.c.) MC-C tumors $\left(650-800 \mathrm{~mm}^{3}\right)$ were surgically excised, leaving intact the underlying skin. In these conditions, recurrent tumors become apparent one week after surgery in $100 \%$ of cases.

b. A model of lung metastases after s.c. LMM3 tumors $\left(650-800 \mathrm{~mm}^{3}\right)$ were radically removed together with the underlying skin when spontaneous metastases were already established in the lung. Local tumors do not re-grow in these conditions, but all mice die with multiple metastases within a month after surgery.

As shown in Figure 3F and G, the growth of local recurrences and metastases was not inhibited by the very same treatments that strongly inhibited the growth of incipient tumors. Actually, the development of local tumor recurrences upon treatment with $\mathrm{ICl}$ was enhanced in the same way as large-sized tumors from which residual tumors were derived by surgical debulking (Figure 3F) - while the growth of metastases was similar in both treated and control groups (Figure 3G).

\section{Simultaneous enhancement of large-sized tumors and inhibition of secondary tumor implants upon treatment with immune-checkpoint inhibitors}

Enhancement of large-sized and residual tumors upon treatment with $\mathrm{ICl}$ and vaccines could be explained, at first sight, by an immunotherapy-mediated down-regulation of the antitumor immune response produced at the local tumor area $(9,32)$. If this were the case, the growth rate of such enhanced tumors could get close to that attained in constitutive immune-deficient nude and NSG mice, but actually, it was significantly higher than in the latter (Suppl. Figure 5). These results suggested that other explanations were necessary. We evaluated the immunologic state of mice bearing large-sized tumors after treatment with $\mathrm{ICl}$ to account for this fact. Tumor-bearing mice produced an anti-tumor immune response (Suppl. Figure 3) but only up to tumor volume reached $500 \mathrm{~mm}^{3}$. Afterwards, such immune response - either significant (for MC-C) or weak (for LMM3) - was sharply down-regulated.

A simple and reliable in vivo marker of anti-tumor immunity is the "concomitant immunity" phenomenon by which tumor-bearing mice are resistant to secondary tumor implants by a specific T-cell dependent mechanism (see Suppl. Figure 3). As shown in Figure 4A-D, upon treatment with anti-CTLA-4 and anti-PDL1, "concomitant immunity" was partially recovered in large-sized tumor-bearing mice. Another marker of anti-tumor immunity, such as tumor-antigen specific splenic T-cell proliferation, was also partially recovered upon treatment (Figure $4 \mathrm{E}-\mathrm{G}$ ). In same way, after ICl therapy, percentage of splenic $\mathrm{CD} 4^{+}$and $\mathrm{CD}^{+} \mathrm{T}$ cells increased $16 \%$ and $32 \%$, respectively, and, reciprocally, $\mathrm{CD}_{2} 5^{+} / \mathrm{FOXP}^{+} \mathrm{T}$ regs dropped $31 \%$.

Consequently, the enhancement of large-sized tumors upon treatment with ICI was, as paradoxical as it could be, associated with an increased anti-tumor immune response. 
The stimulation of tumor growth by a relatively weak immune response has been claimed to be associated with the activation of TLR-4 and p38 pathway in macrophages attracted to the tumor place (16).

Confirming that claim, macrophages collected surrounding large-sized tumors from mice treated with ICI exhibited a significantly higher expression of p38 than that found in similar-sized tumors from nontreated mice or surrounding secondary tumor implants (Figure $4 \mathrm{H}$ and $\mathrm{I}$ ).

\section{Meta-tyrosine and blockade of p38 pathway enable an efficient anti-tumor therapy with anti-tumor vaccines and checkpoint inhibitors}

Acceleration of tumor growth upon vaccines and $\mathrm{ICl}$ treatments might be counteracted by using two different but complementary strategies: a) SB 202190, a selective inhibitor of p38, to counteract the phenomenon of tumor-immune-stimulation; b) meta-tyrosine (m-Tyr), which, according to previously reported results (18) might restrain putative immune-checkpoints not counteracted by classical ICl and, in consequence, to act as an adjuvant for such immunologic therapies. The recovery effect of $\mathrm{m}$-Tyr on splenic T-cell proliferation in immunosuppressed mice is shown in Suppl. Figure 6.

As shown in Figure $5 \mathrm{~A}$ and $\mathrm{B}$, the combined treatment not only counteracted the tumor enhancement effect by $\mathrm{ICl}$ but also achieved significant inhibition of large-sized tumors in both strongly and weakly immunogenic models. Antitumor vaccines produced similar effects to $\mathrm{ICl}$, especially in the strongly immunogenic model. Furthermore, the combined treatment of ICl or vaccines with meta-tyrosine, and SB 202190 was even more effective than classical chemotherapy and radiotherapy against growing murine tumors (Figure 5A). However, despite these promissory results, the tumors continued to grow - although significantly more slowly than controls - and all mice finally reached the experimental endpoint.

A more striking effect was attained against the growth of local recurrences and metastases after surgical debulking the primary tumor. In effect, while local recurrences and residual metastases caused the death of $100 \%$ of non-treated controls or mice treated with each immunologic strategy separately, the combined treatment not only produced tumor-inhibitory effects but even it managed to cure about $80 \%$ of mice bearing local recurrences and about $60 \%$ of mice bearing residual metastases in the lung (Figure $5 \mathrm{C}-\mathrm{F}$ and 6). It is worth noting that, in all the schedules used herein, neither m-Tyr nor SB 202190 produced, on their own, any inhibitory effect but collaborate to make powerfully efficient an otherwise inefficient therapy with vaccines and ICl.

\section{Discussion}

In the last 50-60 years, surgery, radio, and chemotherapy have improved the management of human cancer. However, the progress has been much slower than initially expected, mainly associated with the 
difficulty of treating local recurrent and disseminated cancer. In effect, when a tumor is relatively small, and metastases are absent, cancer could be cured surgically, and 5-years survival rates are estimated at about $90 \%$. On the other hand, when a tumor has not completely excised or it has spread to different sites, those rates often fall below $15 \%$ (33). The true problem is that there is no assurance that a localized cancer will not recur or may be listed as "before metastases" because the number of both remaining local and metastatic tumor cells after surgery may be below the limit of detection by current technology (34).

Limitations of conventional chemotherapeutic drugs to restrain both local recurrent and disseminated tumor cells are associated with its poor aqueous solubility, its lack of tumor-specificity, and the risk of multidrug resistance (35). In this context, immunologic strategies mainly based on the blockade of immune-checkpoints, emerged as a real possibility to treat advanced cancer because they could theoretically overcome the limitations of conventional non-tumor specific anti-cancer therapies.

However, although immune-checkpoint inhibitors (ICI) improved the results achieved with conventional therapies on some clinical tumors, up to date, the overall benefits are supported by a relatively small percentage of patients. Contrastingly, many of them exhibit no significant improvement or even a condition called "hyperprogressive cancer disease" (HPD) with rapid tumor growth, increased metastatic load, and shorter survival times $(10,36)$. It means that, once HPD occurs, ICl are not only invalid for tumor treatment but also detrimental for patients. Across studies, the incidence of HPD among immunotherapy cases ranges from 9-30\%, and some lines of evidence suggest that these rates might have been underestimated $(32,36)$.

Based on these clinical results, it seems to be necessary not only to develop predictors of response to immunotherapy and rational combination therapies that can enhance their efficacy but also to elucidate the mechanisms underlying the phenomenon of HPD.

To this aim, in this work, we have assayed the therapy with anti-CTLA-4 and anti-PD-L1, as well as with classical antitumor vaccines, on two growing murine tumors with widely different degrees of immunogenicity, a strongly immunogenic chemically-induced fibrosarcoma and a weakly immunogenic and highly metastatic mammary adenocarcinoma of spontaneous origin. Treatment was initiated at various stages of tumor growth and assayed on the re-growth of residual tumors (local recurrences and metastases) after surgical tumor extirpation to mimic real clinic situations.

The immunological properties of both tumors, which defined their degree of immunogenicity, were previously elucidated. Pre-treatment with anti-tumor vaccines based on lethally-irradiated tumor cells or dendritic cells (DC) incubated with tumor lysate strongly prevented the challenge with live tumor cells from the chemically-induced fibrosarcoma but not from the spontaneous mammary tumor. To achieve a moderate preventive effect against the latter, tumor lysate and lethally-irradiated tumor cells needed to be pre-treated, with an inhibitor of PD-L1 or PSTAT3. These results suggested that both tumors bore specifictumor antigens. However, in the case of the spontaneous tumor, these antigens were not only hidden but intrinsically weaker than those of the chemically-induced tumor. 
The immunogenic strength of each tumor was also correlated to the antitumor immune response evoked when tumor cells were inoculated into naïve mice. In effect, mice bearing the chemically-induced tumor developed a significant antitumor immune response while mice bearing the spontaneous tumor induced a very low one. In both cases, the immune response was increased upon treatment with anti-tumor vaccines and, more efficiently, with a combination of anti-CTLA-4 plus anti-PD-L1 antibodies. This was reflected in an inhibition of both growing tumors proportionally to tumor immunogenicity.

However, even though vaccines and $\mathrm{ICl}$ were effective in restraining the growth of both strongly and weakly immunogenic growing tumors, they were genuinely efficient only when they faced incipient tumors $\left(<10 \mathrm{~mm}^{3}\right)$. Afterwards, no anti-tumor effects were attained. In fact, when treatment started at the time when tumor was large $\left(>500 \mathrm{~mm}^{3}\right)$, enhancement of tumor growth was achieved in both tumor models. In addition, when treatments were assayed on residual local tumors after surgical large tumor excision, the growth of residual tumors was enhanced in the same way as large-sized tumors from which they were derived. Regarding metastases, neither inhibitory nor stimulatory effects were observed upon treatment. These results indicated that a residual tumor, even composed of a similar number of cells to that of an incipient tumor, behaves, concerning its sensitivity to immunologic strategies, much more like a large than an incipient tumor. As a corollary, data suggest that incipient tumors are not good models to predict the outcome of immunological therapies on residual tumors.

The lack of therapeutic response or even an accelerated growth of non-incipient murine tumors after these immunologic treatments may be paralleled with the lack of response, or the HPD observed in some patients with advanced cancer who have received a therapy with $\mathrm{ICl}$. The mechanisms underlying these undesired therapeutic responses remain speculative. Recent work supports the idea that HPD after therapy with $\mathrm{ICI}$ may be more frequent in patients with MDM2 family amplification and EGFR aberrations than patients without them (37). Another work identified increased expression of oncogenic pathways and mutations in known tumor suppressor genes such as VHL and TSC2 in tumor cells displaying HPD after therapy with anti-PD-1 therapy (38). Others have proposed that, even though treatment with anti-PD1/PD-L1 and anti-CTLA-4 antibodies would usually expand anti-tumor CD8+ and CD4+ T cells, such treatment might, upon certain circumstances, increase the population of PD-1+ T regs producing an effect of immunosuppression. Kamada et al. (39) found that in non-HPD patients, the ratio of Tregs/CD8+ cells, the proportion of Ki67+ T-regs/ Ki67+ CD8+ cells and the percentage of Ki67+ T-regs decreased significantly after nivolumab treatment. At the same time, they remained stable or even reduced in HPD patients. In fact, PD-1+ Tregs and especially M2-like macrophage infiltration induced by anti-PD-1 antibodies have been recently considered a major hallmark of HPD in clinical settings $(10,11)$. In the same line, a correlation between decreased immunogenicity and HPD has been proposed (40).

Although all of the predictors and mechanisms suggested above may play a role in some cases, it is difficult to attribute to them a general role. In effect, in our experiments, differences associated with different genetic backgrounds in the tumor-bearing host are unlikely since all tumor-bearing hosts were inbred mice. In addition, the tumors that displayed hyperprogressive growth upon therapy with $\mathrm{ICI}$ behaved like "normal" tumors (that is, not HPD tumors) when they were transplanted into naïve mice, 
suggesting that no mutations occurred before or during the phase of accelerated tumor growth. In the same way, if a state of immunosuppression were the explanation for the tumor-accelerating effect produced by immunologic strategies on large or local recurrent tumors, the growth of such tumors could get relatively close to that attained in immune-depressed nude and extremely immune-deficient NSG mice but not to grow faster than the latter as it actually occurred. Further, the enhancement of large-sized and residual tumors upon immunological treatments was achieved, surprisingly, in the face of an increased anti-tumor immune response. Lastly, although low immunogenicity may favor HPD in some cases, in this work, accelerated tumor growth after immunologic treatment was observed associated with both strongly and weakly immunogenic tumors.

A putative explanation for the acceleration of tumor growth upon current immunological therapies might be attained on the basis of the theory of tumor-immunostimulation, stated by Prehn many years ago (19). That theory postulates that the antitumor immune response would not be linear, as the orthodoxy predicts, but biphasic with "strong" immune responses producing inhibition, "weak" responses inducing acceleration of tumor growth and "very weak" ones producing no effect (see the Suppl. Figure 1 for a better understanding of the phenomenon). This proposal suggests that immunotherapy against cancer may produce, in the highly immunosuppressive microenvironment of large tumors, weak immune responses that would promote rather than inhibit tumor growth $(16,19,27,41)$. In effect, when tumors have surpassed the critical volume of $500 \mathrm{~mm}^{3}$, tumor-bearing mice usually enter into a state of systemic immune-depression against tumor antigens historically known as "immunological eclipse" that, according to our observations concerning the kinetics of the primary tumor and secondary tumor implants, would be more robust near the primary tumor than anywhere else on the body. That state of immunosuppression is presumably not reversed by incomplete surgical resection because, as we pointed above, recurrences behave, as for their sensitivity to immunologic treatments, much like the large tumors from which they were derived. At that tumor stage, the magnitude of the antitumor immune response near the tumor site could be considered, before any immunologic treatment, as "very weak" and placed near "0" on the biphasic antitumor immune response curve (see Suppl Figure 1). When an immunologic treatment is utilized against these large tumors (and also against local recurrences), it would produce a relatively weak increase of the immune reaction, moving it to the right on the curve, for example toward "c", producing accelerated tumor growth. The observation that metastases from large tumor-bearing mice were neither inhibited nor stimulated upon the very same immunologic treatments that accelerated the primary tumor could be similarly explained by assuming that the state of immunosuppression is less profound far from the primary tumor, where metastases would be established. In consequence, in such places, the basal antitumor immune response would be, for example, near "a" and after the immunologic treatment it would be similarly increased as before, moving the immune response towards "e", where neither inhibitory nor stimulatory effects are expected.

Although antitumor vaccines and $\mathrm{ICI}$ did not produce on their own inhibitory effects on large tumors or their metastases, more stringent strategies, for example by incorporating new and potent adjuvants to the treatment, could move the immune reaction beyond the stimulatory zone up to the inhibitory part of the

Page $14 / 32$ 
curve (for example near " $f$ "). In our experiments this role was achieved by meta-tyrosine (m-Tyr), an unnatural isomer of tyrosine. Former experiments had demonstrated that high concentrations of $\mathrm{m}$-Tyr, chronically administered by the intravenous route, could directly inhibit tumor cell proliferation through inactivation of pSTAT3 and down-regulation of both the NFKB/NOTCH axis and survivin expression (22, 42). More recent experiments demonstrated that $\mathrm{m}$-Tyr, when administered once or few times by the intraperitoneal route, as it was used herein, does not produce any direct antitumor effect but it may boost the overall immune response against different antigens and rescue the organism from states of immunosuppression not counteracted by anti-CTLA-4 and anti-PD-L1 antibodies (18). On this basis, when we combined this schedule of $\mathrm{m}$-Tyr with antitumor vaccines or $\mathrm{ICl}$, a significant inhibitory effect on nonincipient tumors was observed.

Another strategy to overcome the limitations of current immunologic therapies could involve the counteraction of the proper phenomenon of tumor immunostimulation. This phenomenon has recently received a mechanistic interpretation (16) according to which a weak antitumor immune response would promote tumor growth upon enhanced activation of p38 signaling pathway in macrophages recruited at the tumor site. The fact that tumor infiltration by $\mathrm{M} 2$ macrophages is a common finding in clinical cancer displaying HPD after treatment with $\mathrm{ICl}(10)$ further supports the putative involvement of the phenomenon of tumor immunostimulation in those cases. On this basis, when we combined vaccines or $\mathrm{ICl}$ with a specific inhibitor of p38, a significant inhibitory effect on large tumors was observed. In former works $(1,16)$, immunotherapeutic strategies were reported to be improved by the use of non-specific antiinflammatory agents such as indomethacin or low doses of dexamethasone. However, in our assays the anti-inflammatory agent SB202190, specific against p-38, rendered better results.

In our hands, the best therapeutic results were accomplished by combining ICI with both $\mathrm{m}$-Tyr and SB202190 to treat local tumor recurrences and metastases after surgery. This combined therapy produced a profound inhibition of the tumor growth that resulted in $80 \%$ of cures of local recurrent tumors from the strongly immunogenic tumor, and in about $60 \%$ of cures of metastatic residual tumors from the weakly immunogenic one, in a context where, no treatment produced $100 \%$ of deaths in both cases and treatment with $\mathrm{ICl}$ alone produced not only $100 \%$ of deaths but also hyperprogressive or accelerated tumor growth in the case of local recurrent tumors. It is worth to note that the combined therapy utilized in this work was significantly better not only than current immunologic approaches but also than conventional chemotherapy and radiotherapy. The fact that neither $\mathrm{m}$-Tyr nor the specific inhibitor of p38 pathway alone produced any antitumor effect suggested that they did not act on their own but they collaborate with the current immunologic therapies allowing an otherwise ineffective immunologic strategy to have a chance to be effective. In clinical trials for advanced cancer, ICl and other immunologic approaches only evidenced significant beneficial effects in a limited cluster of patients $(43-46)$. We suggested that these patients might exhibit stronger immune reactions than the general population or, alternatively, they have been unable to mount a significant macrophage-related-protumorigenic TLR-4 and p-38 dependent inflammatory response preventing the emergence of a state of immunostimulation. The observation presented in a former paper (16) that the immunostimulatory arm of the immune response curve was not observed in Winn assays carried out in macrophage-depleted and 
TLR-4 knock-out mice, seems to support this suggestion. In fact, the therapeutic antitumor success (when it occurred) of BET (bromo-domain and extra-terminal motif) inhibitors could be associated, at least in part, with their ability to impair macrophage-mediated inflammation (44).

In summary, there is great interest in developing methods and markers that can identify patients and tumor types that could get benefit from different schedules of immunotherapy. In fact, hundreds of trials have been initiated in the last few years and many of them are still ongoing including $\mathrm{ICl}$ or new cancer vaccines either working alone or combined with chemotherapy, radiation therapy, targeted therapy, intratumoral therapy, novel immunomodulators, bispecific and multispecific antibodies, microbioma modulators, adoptive cell therapy including chimeric antigen T-cell receptors and other novel strategies $(45,46)$. In this context, the analysis of genetic profile of tumor antigens, the search for new adjuvants that can blockade new checkpoints not counteracted by already known ICI (m-Tyr is an example of them) and a deeper understanding of the phenomenon of tumor immunostimulation could also contribute to improve the current therapies against cancer.

\section{Abbreviations}

CFSE: Carboxi Fluorescein diacetate Succinimidyl Ester

CICUAL: Committee for the Care and Use of Laboratory Animals

CTLA-4: cytotoxic T-lymphocyte-associated protein

DC: dendritic cells

HPD: hyperprogressive-cancer disease

ICl: immune-checkpoint inhibitors

IR: immune response

m-Tyr: meta-tyrosine

NSG: NOD Scid Gamma

PD-1: programmed cell death-1

PD-L1: PD ligand-1

TD50: Tumor dose 50

TGR: Tumor growth rate

\section{Declarations}




\section{Ethics approval and consent to participate}

All methods were according to NIH Guide for the Care and Use of Laboratory Animals. All experimental protocols were approved by the Committee for the Care and Use of Laboratory Animals (CICUAL) of Instituto de Medicina Experimental (IMEX-CONICET, protocol N005/15). All methods were performed in accordance with ARRIVE guidelines.

\section{Consent for publication}

Not applicable.

\section{Availability of data and materials}

All data generated or analysed during this study are included in this published article and its supplementary information files.

\section{Competing interests}

The authors declare that they have no competing interests.

\section{Funding}

This work was supported by the CONICET (PIP 112201201006 28CO) and the Agencia Nacional de Promoción Científica y Tecnológica, (ANPCyT PICT 1590-2014).

\section{Authors' contributions}

DM performed most of the experiment and helped in writing and correcting the main manuscript text. AD collaborated with the correction of the work and together with PC helped in the accomplishment of some experiments related to figures 1 and 2. BR and MV collaborated with some experiments of figures 1 and 4. OB helped in the design and realization of in vivo experiments. RR designed and supervised all experiments and drafted the manuscript.

\section{Acknowledgements}

We thank the Centro de Investigaciones sobre Porfirinas y Porfirias (CIPYP) of the Hospital de Clínicas for their collaboration with in vivo experiments.

\section{References}

1. Chiarella P, Vulcano M, Bruzzo J, Vermeulen M, Vanzulli S, Maglioco A, et al. Anti-inflammatory pretreatment enables an efficient dendritic cell-based immunotherapy against established tumors. Cancer Immunol Immunother. 2008;57(5):701-18. 
2. Snyder A, Wolchok JD, Chan TA. Genetic basis for clinical response to CTLA-4 blockade. N Engl J Med. 2015;372(8):783.

3. Pardoll DM. The blockade of immune checkpoints in cancer immunotherapy. Nat Rev Cancer [Internet]. 2012;12(4):252-64. Available from: http://dx.doi.org/10.1038/nrc3239

4. Sabado RL, Balan S, Bhardwaj N. Dendritic cell-based immunotherapy. Cell Res. 2017;27(1):74-95.

5. Diesendruck Y, Benhar I. Novel immune check point inhibiting antibodies in cancer therapyOpportunities and challenges. Drug Resist Updat [Internet]. 2017; 30:39-47. Available from: http://dx.doi.org/10.1016/j.drup.2017.02.001

6. Zitvogel L, Kroemer G. Targeting PD-1/PD-L1 interactions for cancer immunotherapy. Oncoimmunology. 2012;1(8):1223-5.

7. Popovic A, Jaffee EM, Zaidi N. Cancers. Emerging strategies for combination checkpoint modulators in cancer immunotherapy. J Clin Invest. 2018;128(8):3209-18.

8. Cruz E, Kayser V. Monoclonal antibody therapy of solid tumors: Clinical limitations and novel strategies to enhance treatment efficacy. Biol Targets Ther. 2019;13:33-51.

9. Champiat S, Dercle L, Ammari S, Massard C, Hollebecque A, Postel-Vinay S, et al. Hyperprogressive disease is a new pattern of progression in cancer patients treated by anti-PD-1/PD-L1. Clin Cancer Res. 2017;23(8):1920-8.

10. Zang H, Peng J, Zheng H, Fan S. Hyperprogression After Immune-Checkpoint Inhibitor Treatment: Characteristics and Hypotheses. Front Oncol. 2020;10(April):1-7.

11. Russo G Lo, Moro M, Sommariva M, Cancila V, Boeri M, Centonze G, et al. Antibody-Fc/FcR interaction on macrophages as a mechanism for hyperprogressive disease in non-small cell lung cancer subsequent to PD-1/PD-L1 blockade. Clin Cancer Res. 2019;25(3):989-99.

12. Kon E, Benhar I. Immune checkpoint inhibitor combinations: Current efforts and important aspects for success. Drug Resist Updat. 2019;45(July):13-29.

13. Hewitt HB, Blake ER WA. A critique of the evidence for active host defence against cancer based on personal studies of 27 murine tumors of spontaneous origin. Br J Cancer. 1976;33: 241-59.

14. Zheng L, Zhou B, Meng X, Zhu W, Zuo A, Wang X, et al. A model of spontaneous mouse mammary tumor for human estrogen receptor- and progesterone receptor-negative breast cancer. Int $\mathrm{J}$ Oncol. 2014;45(6):2241-9.

15. Meuwissen R, Berns A. Mouse models for human lung cancer. Genes Dev. 2005;19(6):643-64.

16. Chiarella $P$, Chiarella $P$, Vermeulen $M$, Vermeulen $M$, Montagna DR, Vallecorsa $P$, et al. improvement of antitumor Therapies Based on Vaccines and immune-checkpoint inhibitors by counteracting Tumorimmunostimulation. Front Oncol. 2018;8(86).

17. Mermod M, Hiou-Feige A, Bovay E, Roh V, Sponarova J, Bongiovanni M, et al. Mouse model of postsurgical primary tumor recurrence and regional lymph node metastasis progression in HPVrelated head and neck cancer. Int J Cancer. 2018;142(12):2518-28. 
18. Montagna DR, Duarte A, Todero MF, Ruggiero RA, Isturiz M, Rearte B. Meta-tyrosine modulates the immune response induced by bacterial endotoxins. Immunobiology [Internet]. 2020;225(1):151856. Available from: https://doi.org/10.1016/j.imbio.2019.10.005

19. Prehn RT. The immune reaction as a stimulator of tumor growth. Science (80- ). 1972;176(4031):170-1.

20. Ichim C V. Revisiting immunosurveillance and immunostimulation: Implications for cancer immunotherapy. J Transl Med. 2005;3:1-13.

21. Franco M, Bustuoabad OD, Di Gianni PD, Goldman A, Pasqualini CD, Ruggiero RA. A serum-mediated mechanism for concomitant resistance shared by immunogenic and non-immunogenic murine tumours. Br J Cancer. 1996;74(2):178-86.

22. Ruggiero RA, Bruzzo J, Chiarella P, Di Gianni P, Isturiz MA, Linskens S, et al. Tyrosine isomers mediate the classical phenomenon of concomitant tumor resistance. Cancer Res. 2011;71(22):7113-24.

23. Colombo LL, Vanzulli SI, Villanueva A, Cañete M, Juarranz A, Stockert JC. Long-term regression of the murine mammary adenocarcinoma, LM3, by repeated photodynamic treatments using mesotetra (4-N-methylpyridinium) porphine. Int J Oncol. 2005;27(4):1053-9.

24. Nestle FO, Alijagic S, Gilliet M, Sun Y, Grabbe S, Dummer R, et al. Vaccination of melanoma patients with peptide- or tumor lysate-pulsed dendritic cells. Nat Med. 1998 Mar;4(3):328-32.

25. Inaba K, Inaba M, Romani N, Aya H, Deguchi M, Ikehara S, et al. Generation of large numbers of dendritic cells from mouse bone marrow cultures supplemented with granulocyte/macrophage colony-stimulating factor. J Exp Med. 1992 Dec 1;176(6):1693-702.

26. Ruggiero RA, Bustuoabad OD, Bonfil RD, Meiss RP, Pasqualini CD. "Concomitant Immunity" in Murine Tumours of Non-Detectable Immunogenicity. Br J Cancer. 1985;51(1):37-48.

27. Chiarella P, Reffo V, Bruzzo J, Bustuoabad OD, Ruggiero RA. Therapeutic anti-tumor vaccines: From tumor inhibition to enhancement. Clin Med Oncol. 2008;2:237-45.

28. Manthey CL, Wang SW, Kinney SD, Yao Z. SB202190, a selective inhibitor of p38 mitogen-activated protein kinase, is a powerful regulator of LPS-induced mRNAs in monocytes. J Leukoc Biol. 1998;64(3):409-17.

29. Fujita M, Zhu X, Sasaki K, Ueda R, Low KL, Pollack IF, et al. Inhibition of STAT3 Promotes the Efficacy of Adoptive Transfer Therapy Using Type-1 CTLs by Modulation of the Immunological Microenvironment in a Murine Intracranial Glioma. J Immunol. 2008;180(4):2089-98.

30. Zhu H, Bengsch F, Svoronos N, Rutkowski MR, Benjamin G, Allegrezza MJ, et al. BET Bromodomain Inhibition Promotes Anti-Tumor Immunity by Suppressing PD-L1 expression. Cell Rep. 2017;16(11):2829-37.

31. Spranger S, Koblish HK, Horton B, Scherle PA, Newton R, Gajewski TF. Mechanism of tumor rejection with doublets of CTLA-4, PD-1/PD-L1, or IDO blockade involves restored IL-2 production and proliferation of CD8+ T cells directly within the tumor microenvironment. J Immunother Cancer. 2014;2(1):1-14.

32. Popat S. Hyperprogression with immunotherapy: Is it real? Cancer. 2019;125(8):1218-20. 
33. Chen J, Sprouffske K, Huang Q, Maley CC. Solving the puzzle of metastasis: The evolution of cell migration in neoplasms. PLoS One. 2011;6(4).

34. DR M, P C, RP M, RA R. The acceleration of metastases after tumor removal and the paradoxical phenomenon of concomitant tumor resistance. J Cancer Res Ther. 2018;6(6):41-51.

35. Moorthi C, Manavalan R, Kathiresan K. Nanotherapeutics to overcome conventional cancer chemotherapy limitations. J Pharm Pharm Sci. 2011;14(1):67-77.

36. Besse B, Ferrara R, Mezquita L, Texier M, Lahmar J, Audigier-Valette C, et al. Hyperprogressive Disease in Patients with Advanced Non-Small Cell Lung Cancer Treated with PD-1/PD-L1 Inhibitors or with Single-Agent Chemotherapy. JAMA Oncol. 2018;4(11):1543-52.

37. Kato S, Goodman A, Walavalkar V, Barkauskas DA, Sharabi A, Kurzrock R. Hyperprogressors after immunotherapy: Analysis of genomic alterations associated with accelerated growth rate. Clin Cancer Res. 2017;23(15):4242-50.

38. Xiong D, Wang Y, Singavi AK, Mackinnon AC, George B, You M. Immunogenomic Landscape Contributes to Hyperprogressive Disease after Anti-PD-1 Immunotherapy for Cancer. iScience [Internet]. 2018; 9:258-77. Available from: https://doi.org/10.1016/j.isci.2018.10.021

39. Kamada T, Togashi Y, Tay C, Ha D, Sasaki A, Nakamura Y, et al. PD-1+ regulatory T cells amplified by PD-1 blockade promote hyperprogression of cancer. Proc Natl Acad Sci U S A. 2019;116(20):999910008.

40. Faure M, Rochigneux P, Olive D, Taix S, Brenot-Rossi I, Gilabert M. Hyperprogressive disease in anorectal melanoma treated by PD-1 inhibitors. Front Immunol. 2018;9(APR):1-6.

41. Gueron G, Anselmino N, Chiarella P, Ortiz EG, Vickers SL, Paez A V, et al. Game-changing restraint of Ros-damaged phenylalanine, upon tumor metastasis. Cell Death Dis. 2018;9.

42. Prehn RT, Prehn LM. Cancer immunotherapy by immunosuppression. Theor Biol Med Model. 2010;7(1):1-9.

43. Parmiani G, Maccalli $C$. The early antitumor immune response is necessary for tumor growth: Revisiting prehn's hypothesis in the human melanoma system. Oncoimmunology. 2012;1(6):930-4.

44. Belkina AC, Nikolajczyk BS, Denis G V. BET Protein Function Is Required for Inflammation: Brd2 Genetic Disruption and BET Inhibitor JQ1 Impair Mouse Macrophage Inflammatory Responses. J Immunol. 2013;190(7):3670-8.

45. Meric-Bernstam F, Larkin J, Tabernero J, Bonini C. Enhancing anti-tumour efficacy with immunotherapy combinations. Lancet [Internet]. 2021;397(10278):1010-22. Available from: http://dx.doi.org/10.1016/S0140-6736(20)32598-8

46. Mercedes Segovia, Sofa Russo, Mathias Jeldres, Yamil Mahmoud, Valentina Perez, et al. Targeting TMEM176B Enhances Antitumor Immunity and Augments the Efficacy of Immune Checkpoint Blockers by Unleashing Inflammasome Activation. Cancer Cell 2019;35 (5):767-781.

\section{Figures}


A

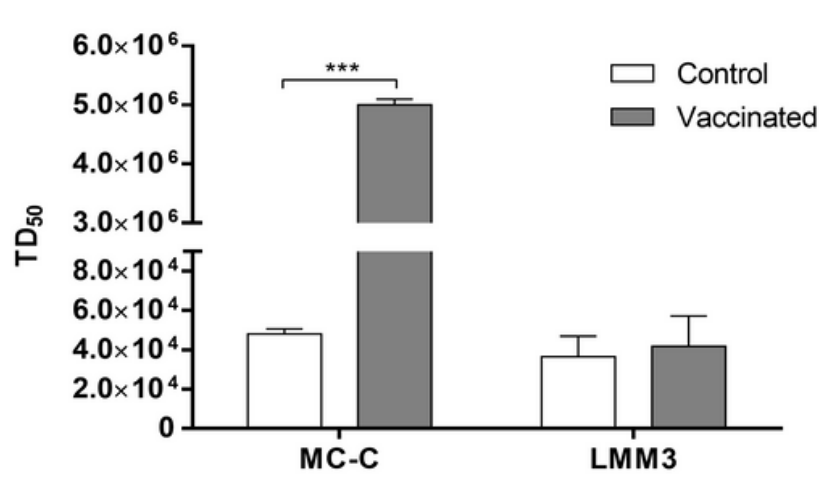

C

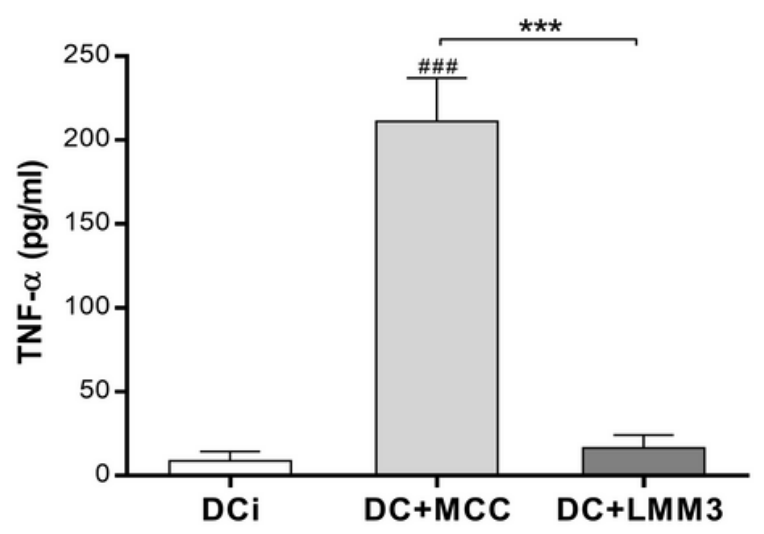

$\mathbf{E}$

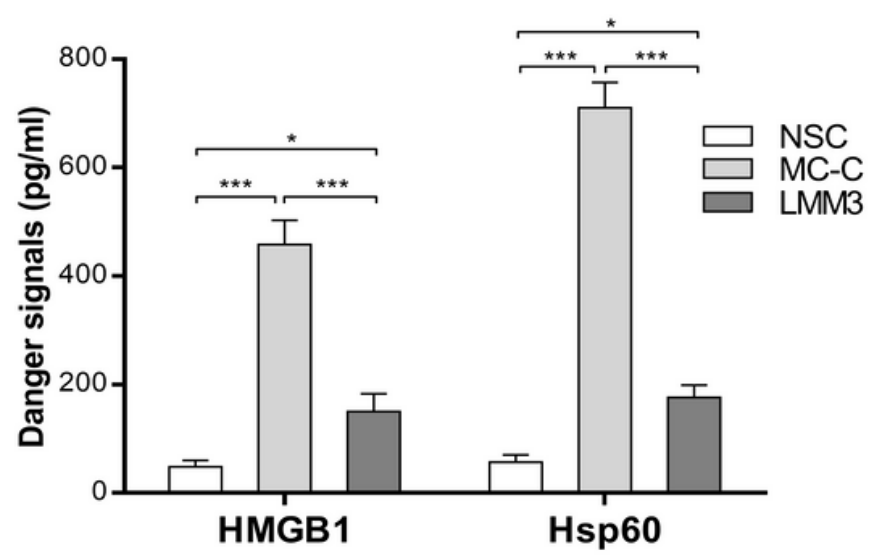

B

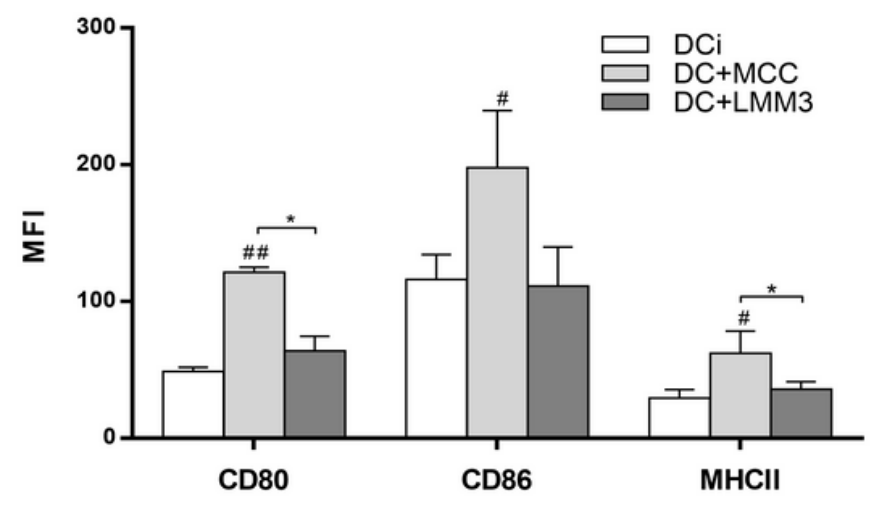

D

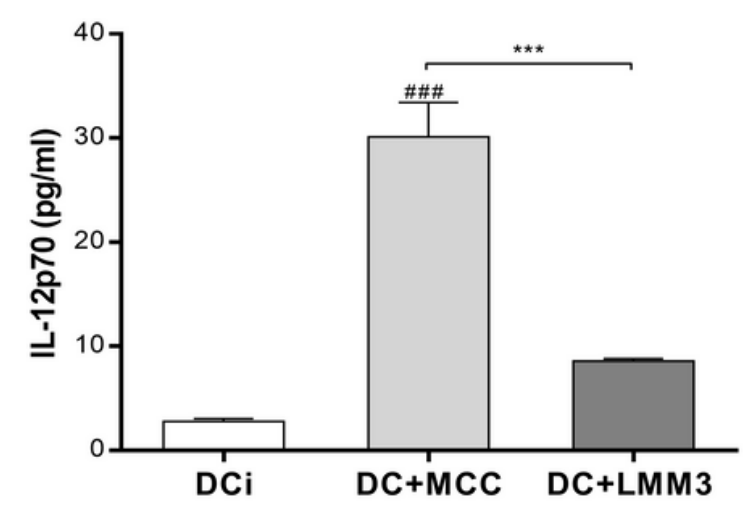

$\mathbf{F}$

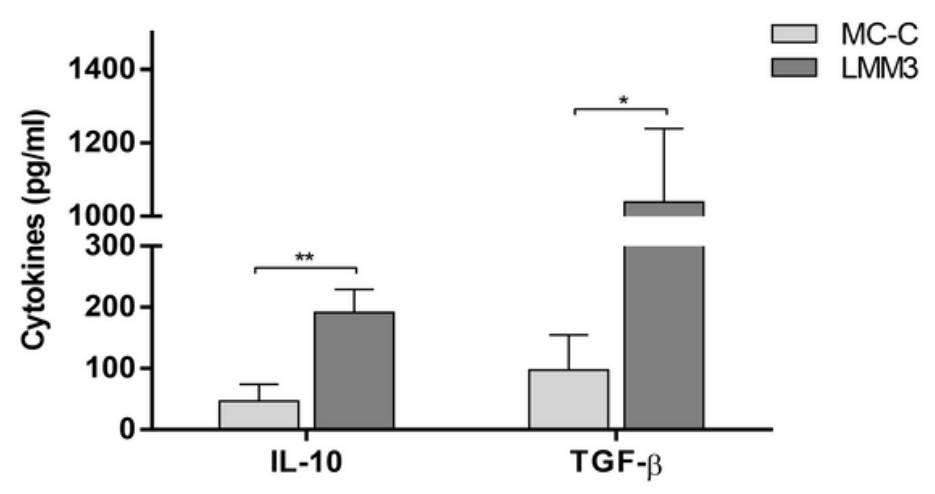

Figure 1

Immunologic properties of MC-C and LMM3 tumors. (A): Vaccinating capacity against MC-C or LMM3 of DC stimulated with MC-C or LMM3 lysates, respectively. Two doses of DC of different groups were inoculated in the footpad of mice 14 and 7 days before the s.c. challenge with different doses of tumor cells. The vaccinating capacity was measured as an increase of TD50 of tumors in treated mice compared to control untreated mice. Data represent the mean \pm SEM of two independent experiments. In each experiment, 20-25 mice per group were utilized. Similar results were obtained when lethallyirradiated tumor cells were used as a vaccination strategy. (B): Markers of DC maturation stimulated with 
lysates from MC-C and LMM3 tumors. Expression of cell-surface receptors CD80, CD86, and MHCll was evaluated by flow cytometry. Controls were unstimulated DC (DCi). DC stimulated with lysate of normal spleen cells displayed similar results to that obtained with $\mathrm{DCi}$, and, for simplicity, they were omitted. MFI $=$ Mean fluorescence intensity. Data represent the mean \pm SEM of two independent experiments. (C, D): Concentration of TNF-a (C) and IL-12p70 (D) ( $\mathrm{pg} / \mathrm{ml})$ in supernatants of DC stimulated with MC-C or LMM3 lysates. Data represent the mean \pm SEM of three independent experiments. (E) Concentration of danger signals HGMB1 and Hsp 60 (pg/ml) in lysates from MC-C and LMM3 tumors and in a lysate prepared from normal spleen cells (NSC). Each value represents the mean \pm SEM of three assays. (F) Concentration of cytokines IL-10 and TGF- $\beta$ in lysates from MC-C and LMM3 tumors. Levels of IL-10 and TGF- $ß$ in NSC were undetectable. Each value represents the mean \pm SEM of three assays. ELISA assays evaluated cytokines and danger signals levels. Statistical comparison between: experimental groups vs. control (DCi): \# $p<0.05 ; \# \# p<0.01$; \#\#\# $<<0.001$. Statistical comparison among the experimental groups: * $p<0.05 ; * \star p<0.01 ; * \star \star ~ p<0.001$. 

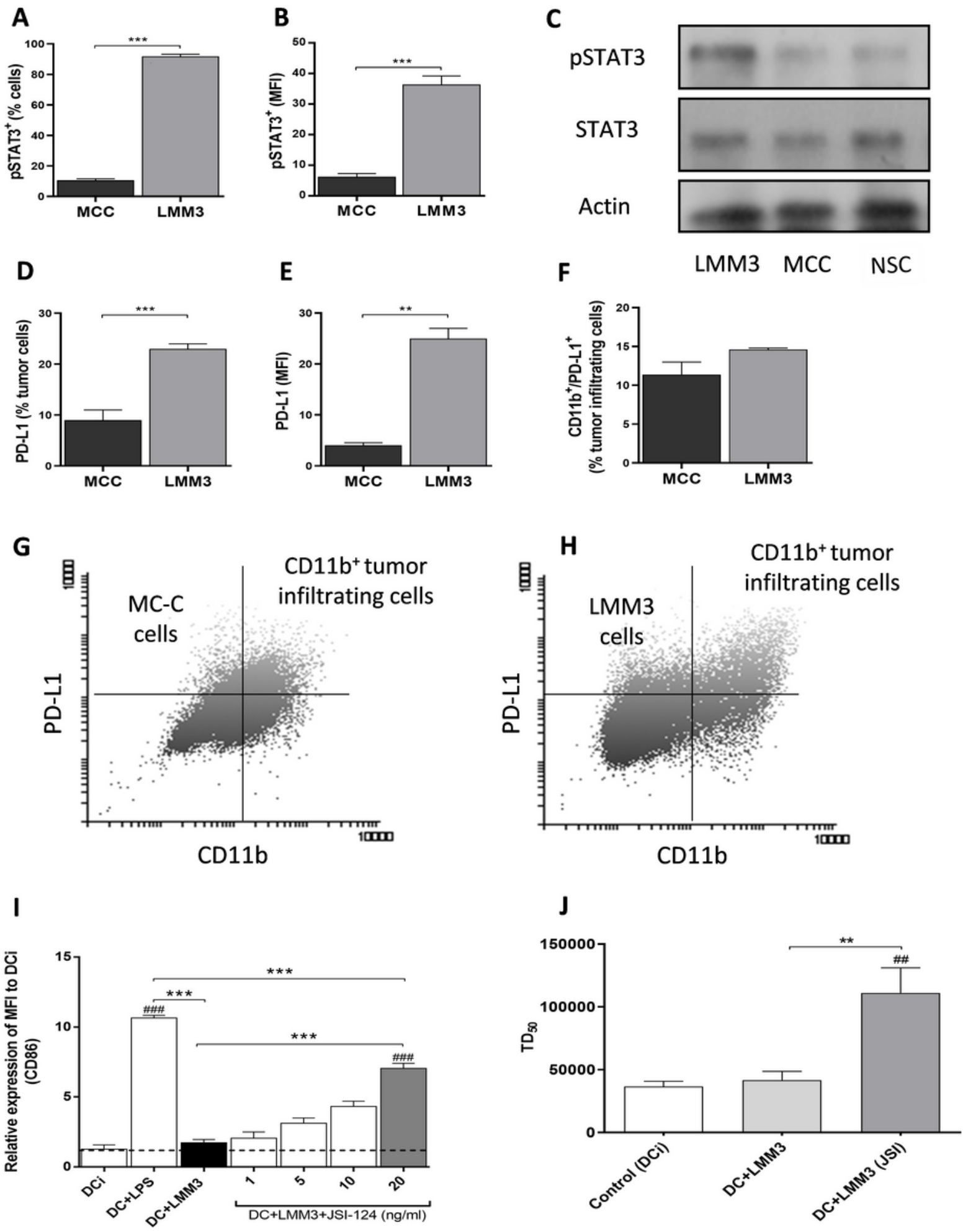

\section{Figure 2}

Expression of activated STAT3 (pSTAT3) and PD-L1 in MC-C and LMM3 tumors. Percentage of cells pSTAT3+ (A) and mean fluorescent intensity (MFI) (B) were evaluated by flow cytometry. pSTAT3 was determined by Western Blotting in MC-C, LMM3 tumor cells, and normal splenocytes (NSC). Controls with actin and total STAT3 were added (C). Results are representative of three similar experiments. (D, E, F) Expression of PD-L1+ in MC-C, LMM3 and myeloid infiltrating cells. Percentage of cells PD-L1+ (D, F) and 
MFI (E). Each value represents the mean \pm SEM of three assays. $(G, H)$ Representative dot plots of expression of PD-L1 in MC-C and LMM3 tumor cells and CD11b+tumor-infiltrating cells. (I) Acquired capacity of LMM3 lysate to promote the maturation of DC by pre-treatment of LMM3 tumor cells with an inhibitor of STAT3, called JSI-124. DC were incubated with LMM3 lysate or with lysate from LMM3 cells that had been pre-treated in vitro for 24 hours with different concentrations $(1,5,10$, and $20 \mathrm{ng} / \mathrm{ml})$ of JSI-124. DC incubated with LPS served as a positive control. Negative controls were immature DC (DCi). Data represent the mean \pm SEM of three independent experiments. $(J)$ Vaccinating capacity against LMM3 of DC stimulated with a lysate from LMM3 cells that had been treated in vitro with $20 \mathrm{ng} / \mathrm{ml}$ of JSI-124. Two doses of DC of the different groups were inoculated in the footpad of mice 14 and 7 days before the s.c. challenge with different doses of LMM3 tcells. Vaccinating capacity was measured as an increase of DT50 of LMM3 tumor in treated mice compared to control. Data represent the mean \pm SEM of two independent experiments. In each experiment, 20-25 mice per group were utilized. Statistical comparison between experimental groups and DCi: \#\#p<0.01; \#\#\#p<0.001. Statistical comparison among experimental groups: $* * p<0.01 ; * \star *<0.001$. 
A

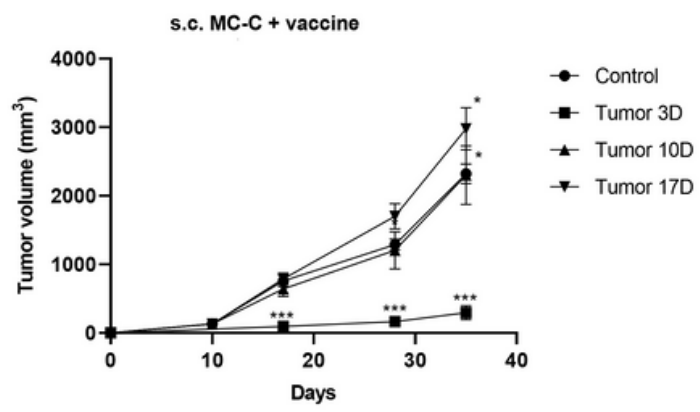

C

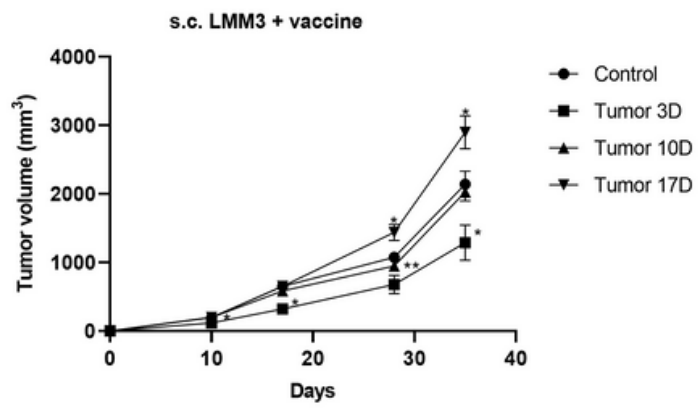

B

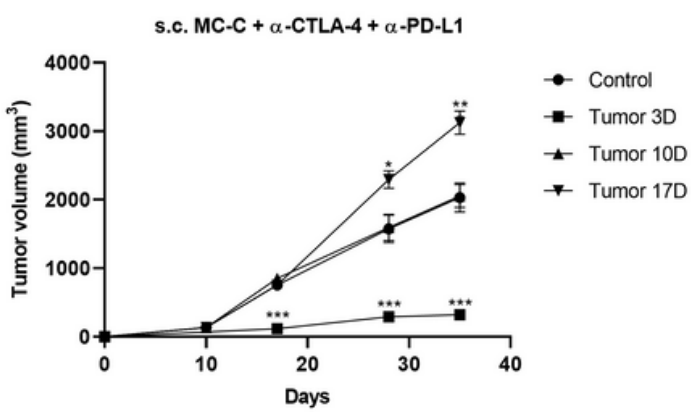

D

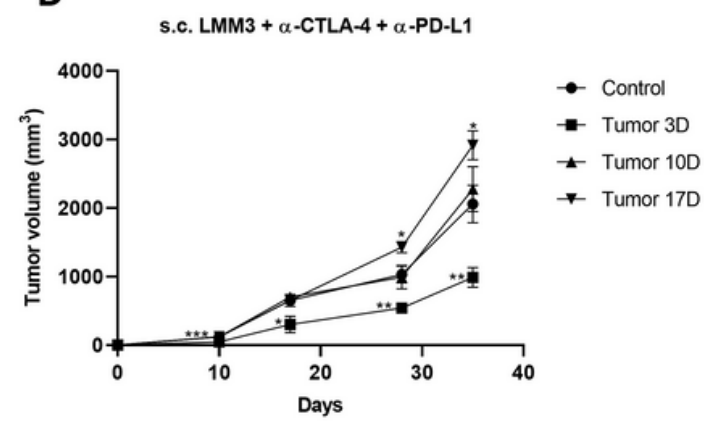

$\mathbf{E}$

Treatment of LMM3 metastases with $\alpha-C$ TLA- $4+\alpha-$ PD-L1
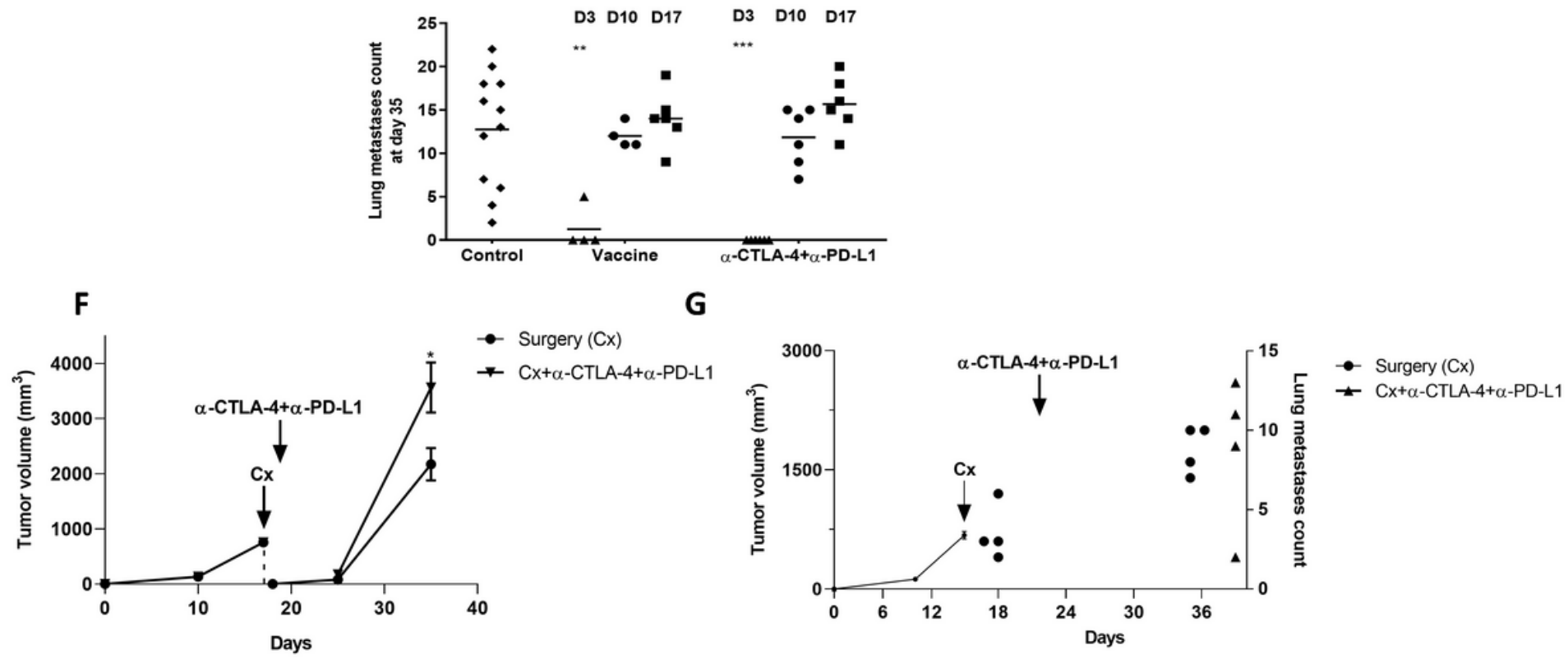

Figure 3

Therapeutic antitumor immunological schedules against growing and residual tumors. (A, C) Antitumor vaccines. 1×105 MC-C (A) or LMM3 (C) cells were inoculated s.c. in the right flank. DC were incubated with LMM3 lysate or with lysate from LMM3 cells that had been pre-treated in vitro for 24 hours with different concentrations $(1,5,10$, and $20 \mathrm{ng} / \mathrm{ml})$ of JSI-124. DC incubated with LPS served as a positive control. Negative controls were immature DC (DCi). Mice received in the left flank an antitumor vaccine (DC stimulated in vitro with MC-C lysate (A) or DC stimulated in vitro with LMM3 lysate from cells that had been treated in vitro with $20 \mathrm{ng} / \mathrm{ml}$ of JSI-124 (C)), starting at day 3, 10 or 17 of tumor growth. (B, D). 
ICl. 1×105 MC-C (B) or LMM3 (D) cells were inoculated s.c. in the right flank. Afterward, mice received anti-CTLA-4 + anti-PD-L1, starting at day 3, 10, or 17 of tumor growth. For simplicity, groups treated with anti-CTLA-4 alone and anti-PD-L1 alone were omitted. (E) Effect of vaccines and ICl on the growth of lung metastases of LMM3. Mice were treated with anti-CTLA-4 + anti-PD-L1, starting at day 3, 10, or 17 of tumor growth. Groups of mice that did not receive any treatment served as controls. $(F, G)$ Therapeutic antitumor immunological schedules in MC-C local recurrences $(F)$ and postsurgical LMM3 lung metastases (G). Different groups of mice received anti-CTLA-4 + anti-PD-L1, starting the day after surgery. Each dose of anti-CTLA-4 and anti-PD-L1 was $100 \mu \mathrm{g}$. Anti-CTLA-4 was inoculated three times a week and anti-PD-L1 for 9 consecutive days, both i.p. Data from Figures A, B, C, D, F, and G represent the mean \pm SEM of two or three independent experiments. 4 - 6 mice per group were utilized. Statistical comparison between experimental groups and control: ${ }^{*} p<0.05 * * ; p<0.01$; $* \star * ; p<0.001$. 
A

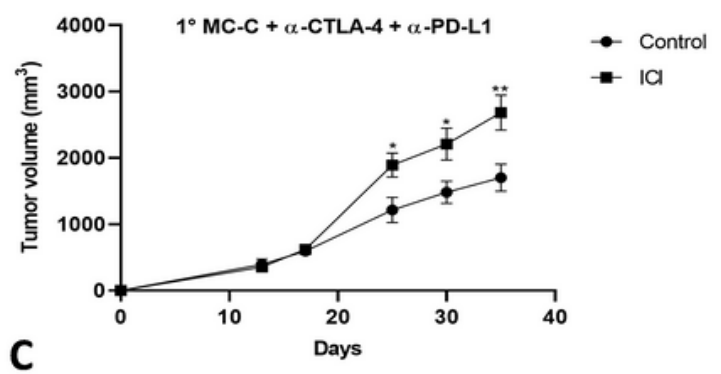

$$
\text { C }
$$
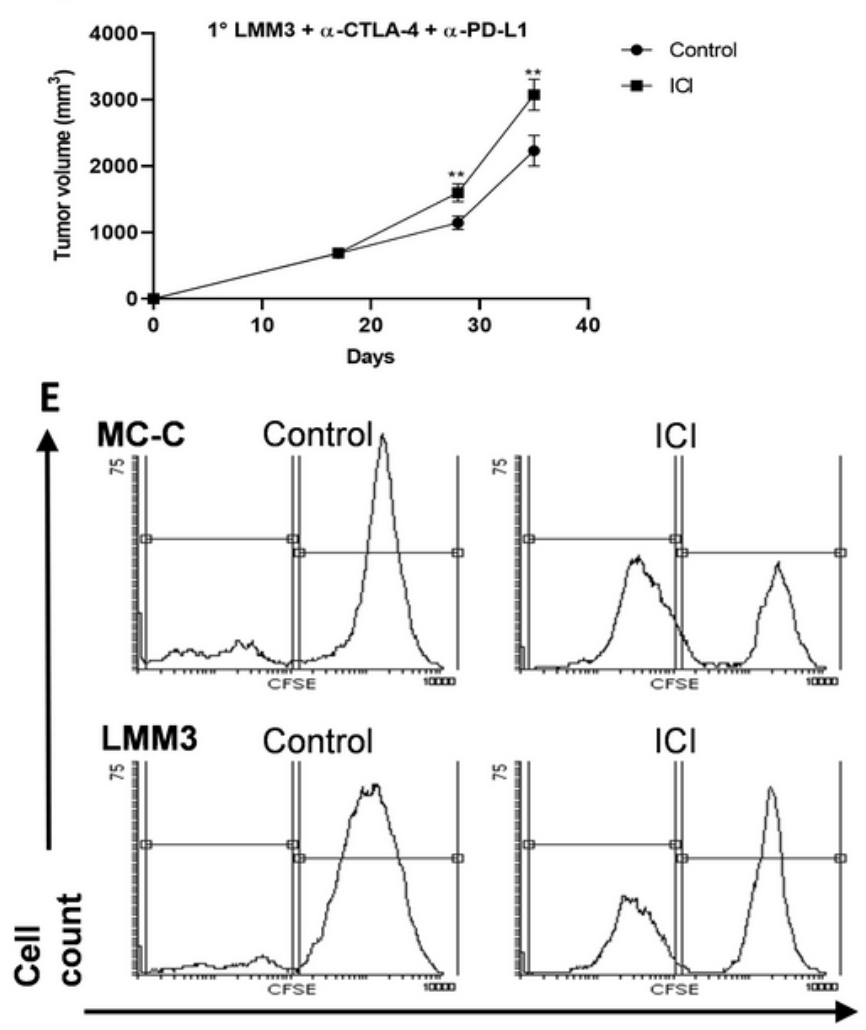

H

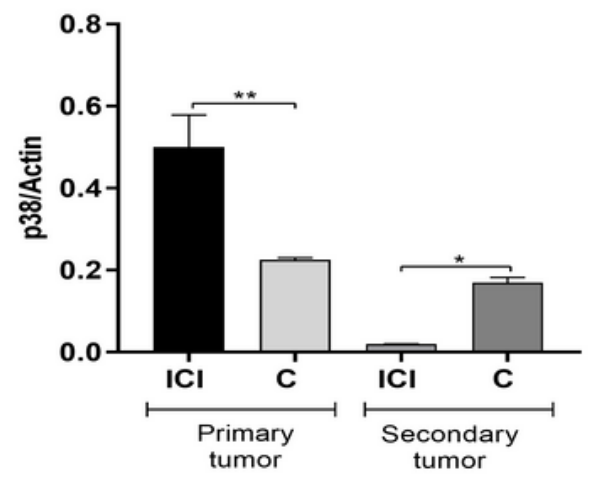

B
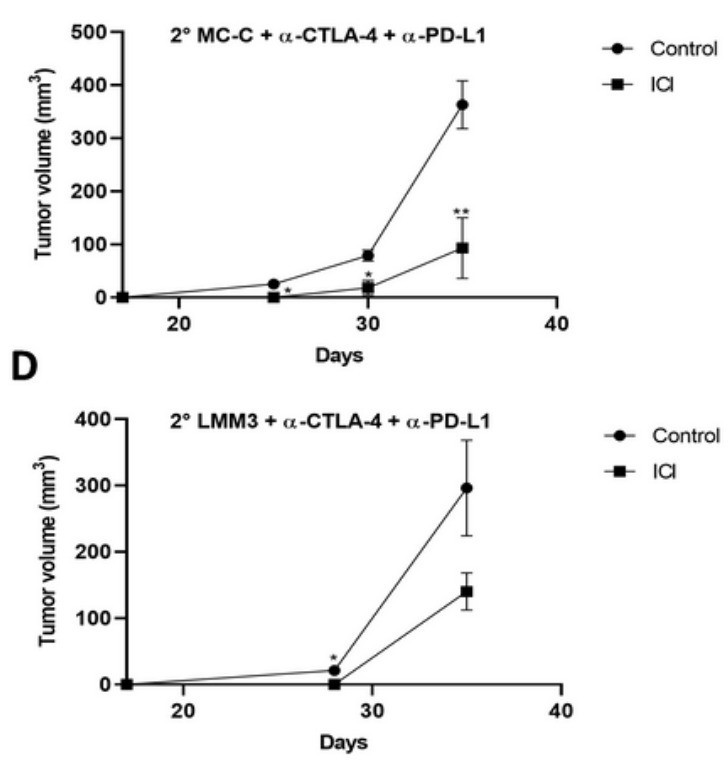

F $\quad$ MC-C

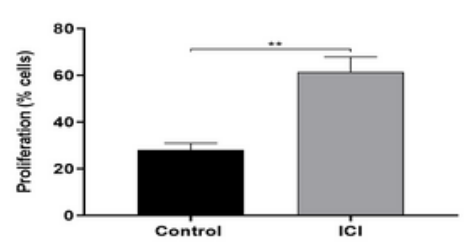

G LMM3

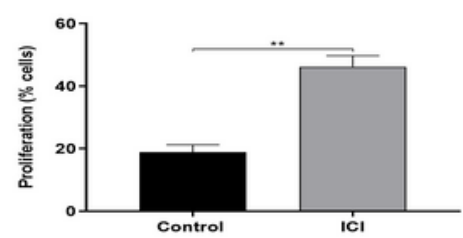

I

p38

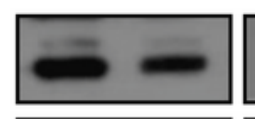

Actin
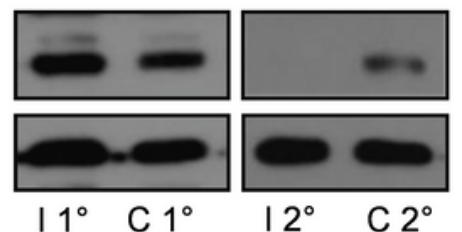

\section{Figure 4}

Simultaneous effects of immune checkpoint inhibitors (ICI) on primary large-sized tumors and secondary tumor-bearing mice. (A, B, C, D) Mice bearing MC-C (A) or LMM3 tumor (C) measuring 800 mm3 were challenged at day 17 with a secondary tumor implant $(B, D)$ carried out in the contralateral flank and, simultaneously, treated with ICI (anti-CTLA-4 + anti-PD-L1). 5×105 tumor cells were inoculated for MC-C and LMM3 primary tumors, and 2×105 tumor cells for secondary implants. For each tumor, the figure 
shows a representative experiment ( $n=4-6$ mice per group) out of two experiments that rendered similar results. Data were expressed as mean $(\mathrm{mm} 3) \pm$ SEM of tumor volume. (E) Representative CFSE flow cytometric histograms of splenic T-cells from MC-C and LMM3 primary and secondary tumors bearing mice. $(F, G)$ Percentage of the proliferation of splenic T cells from MC-C (F) and LMM3 $(G)$ primary and secondary tumors bearing mice. Mice were treated with anti-CTLA-4 + anti-PD-L1 (immunized group), and non-treated mice served as control (control group). Each value represents the mean \pm SEM of two assays. Each dose of anti-CTLA-4 and anti-PD-L1 was $100 \mu \mathrm{g}$ per mouse. For simplicity, groups treated with antiCTLA-4 alone and anti-PD-L1 alone were omitted. $(H, I)$ Expression of phosphorylated (p)-38 (p38) by Western blotting. Macrophages $(3 \times 106$ cells) were collected surrounding the s.c. primary and secondary MC-C tumors 7 days post-secondary implant. Mice were treated with anti-CTLA-4 + anti-PD-L1 (immunized group), and non-treated mice served as control. A representative experiment is shown. The figure shows levels of p38 in the different groups, normalized with beta-actin densitometric units, representing the mean \pm SE of three independent experiments. Statistical comparison between experimental groups and control: * $p<0.02 ;{ }^{* \star} p<0.01 ; * \star \star p<0.001$. 

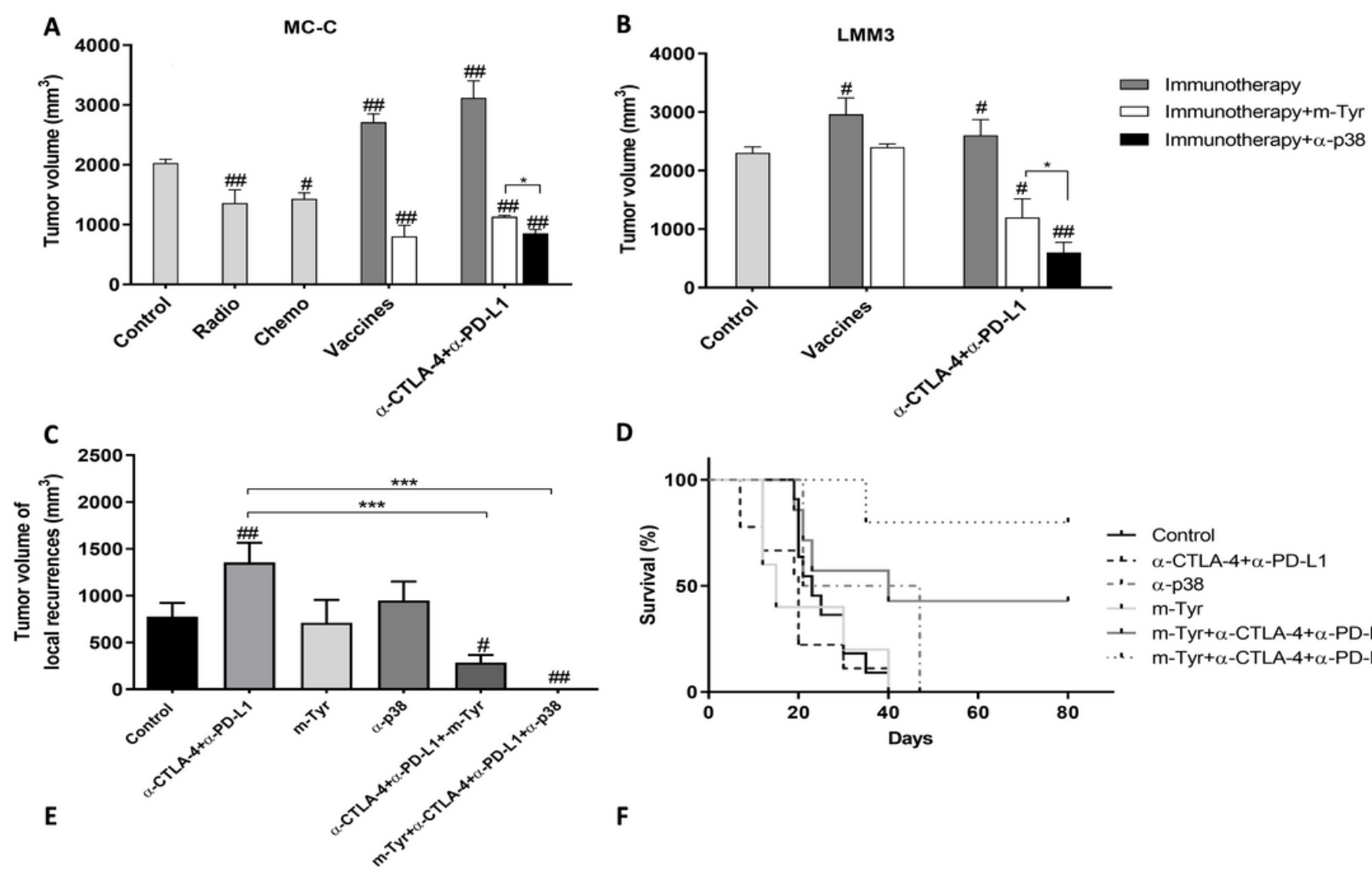

D

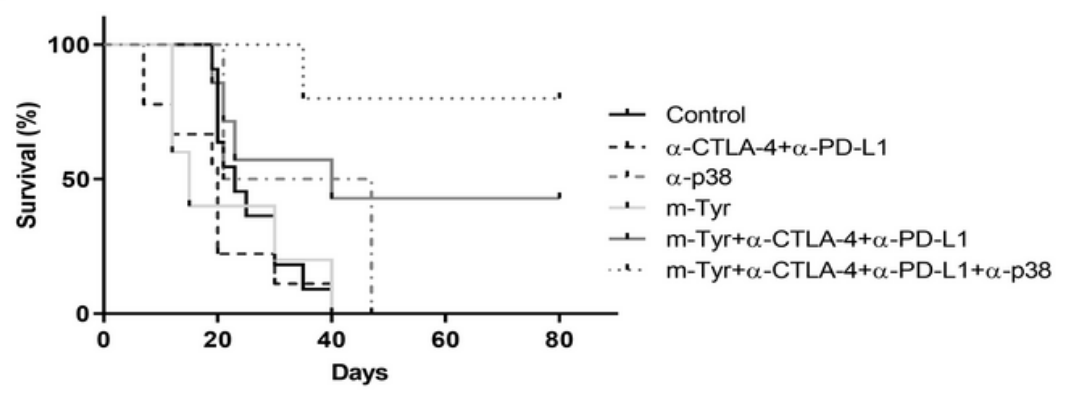

$\mathbf{F}$
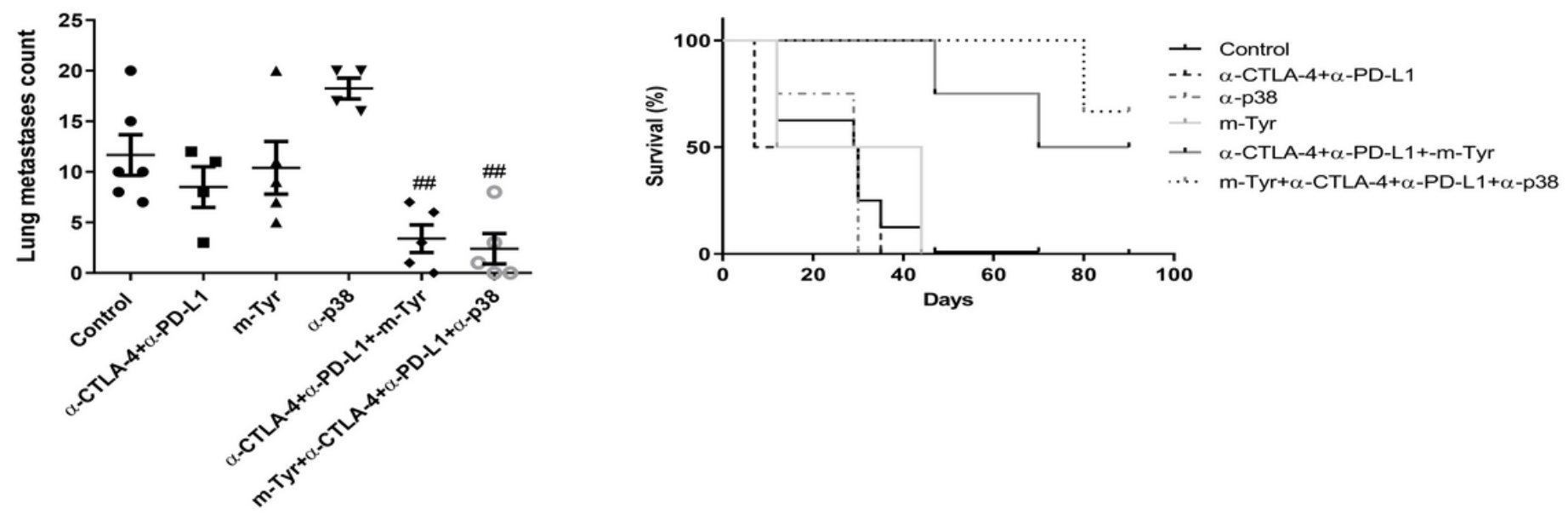

Figure 5

Effect of combined immunological treatment on growing and residual tumors. (A, B) $1 \times 105$ MC-C (A) or LMM3 (B) cells were inoculated s.c. in the right flank. On day 17 of tumor growth, mice received immunotherapy [anti-CTLA-4 + anti-PD-L1, or antitumor vaccines (same schedules to figure 3)], immunotherapy plus m-Tyr (1.5 mg i.p. one dose, day 18) or immunotherapy plus anti-p38 (SB202190: $0.5 \mathrm{mg} / \mathrm{kg}$ i.p. for four days starting at day 18 of tumor growth). For MC-C, mice received a dose of radiotherapy (2000 grades) or vincristine $(1 \mathrm{mg} / \mathrm{kg}$ i.p.). Tumor-bearing mice without treatment served as control. Tumor volumes were measured at day 35. (C, D, E, F). Effect of combined immunotherapy on local recurrences and metastases. Model of MC-C local recurrences: s.c. MC-C tumors (about $800 \mathrm{~mm} 3$ ) were surgically excised, leaving underlying skin. Model of lung metastases: s.c. LMM3 tumors (about 800 
$\mathrm{mm} 3$ ) were radically removed with underlying skin when spontaneous metastases are already established. Mice were treated with immunotherapy (anti-CTLA-4 + anti-PD-L1 (E, F), immunotherapy plus m-Tyr, immunotherapy plus SB202190 (on suture line for MC-C or i.v for LMM3) or immunotherapy plus both $\mathrm{m}$-Tyr and SB202190. Operated mice without treatment served as control. Tumor volumes were evaluated two weeks after surgery (D, F). Survival percent were assessed 90 days after surgery. Data represent \pm SEM of tumor volume $(\mathrm{mm} 3)$ of 8 - 12 mice per group. For local recurrences and residual metastases, a sub-group was sacrificed 15 days after surgery to histological analysis and to count number of metastases. In all cases, the effects of treatments with m-Tyr or SB202190 alone were similar to control group and omitted for simplicity. Statistical comparison between experimental groups and control: \# $p<0.05 ; \# \#$ : $p<0.01$. Statistical comparison among experimental groups: ${ }^{*} p<0.05 ; \star \star * ; p<$ 0.001 . 

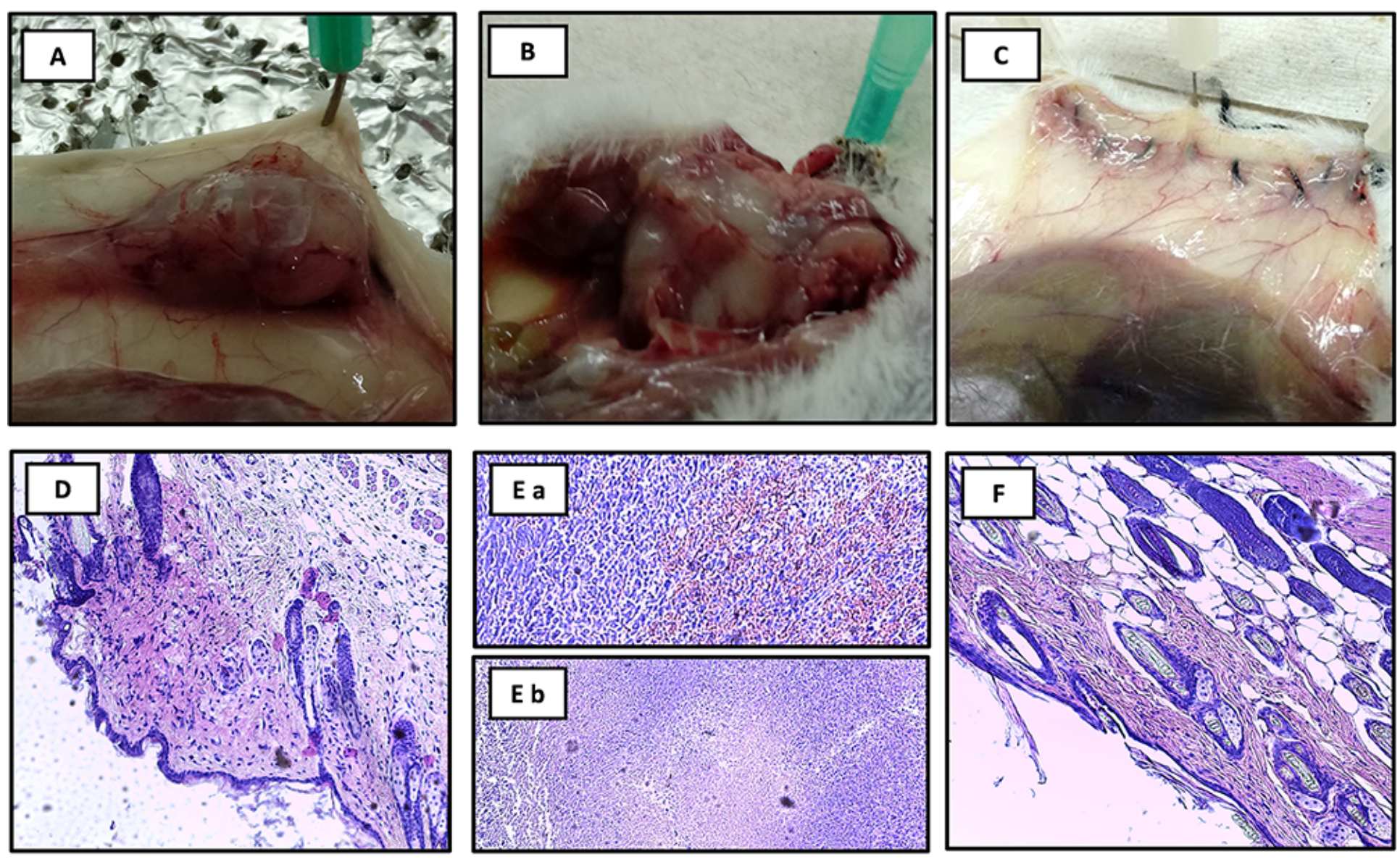

\section{LMM3 postsurgical lung metastases}
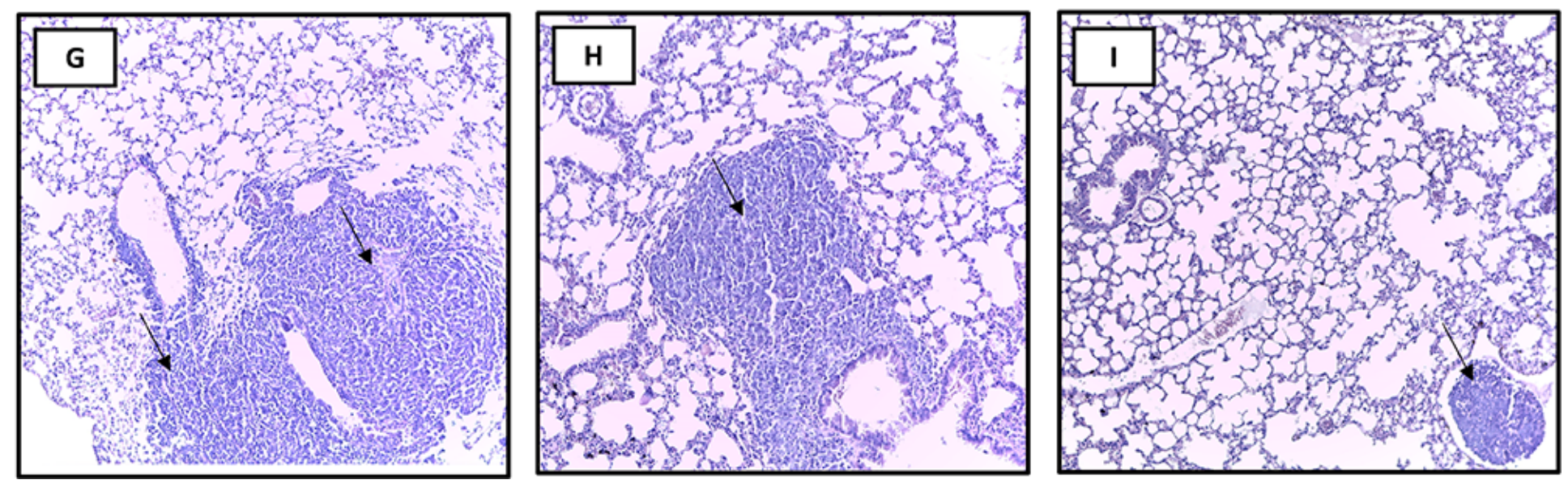

Figure 6

Representative macroscopic and histopathological images of tumor recurrences. (A - F) MC-C tumor local recurrences images (H\&E staining, 100X) at day 15 post-surgical removal, corresponding to untreated control (A, D), anti-CTLA-4 + anti-PD-L1 (B, E) and anti-CTLA-4+anti-PD-L1 + m-Tyr + anti-p38 (C, F). Noted the medium-size tumor of untreated mouse $(A)$, the large size tumor of a mouse treated with anti-CTLA4+anti-PD-L1 (B), and an imperceptible tumor mass in the suture line from a mouse treated with anti- 
CTLA-4 +anti-PD-L1+ m-Tyr + anti-p38 (C). (G - I) LMM3 post-surgical lung metastases images (H\&E staining, 100X) at day 15 after surgery, corresponding to control (G), anti-CTLA-4 + anti-PD-L1 (H) and anti-CTLA-4 + anti-PD-L1 + m-Tyr + anti-p38 (I) treated mice. Arrows point sites of tumor cells in the same field.

\section{Supplementary Files}

This is a list of supplementary files associated with this preprint. Click to download.

- Additionalfiles.docx

- SupplementaryFigures.pdf 